\title{
Impact of Automated Truck Platooning on the Performance of Freeway Mixed Traffic Flow
}

\author{
Seolyoung Lee $\mathbb{D}^{1},{ }^{1}$ Cheol Oh $\mathbb{D},{ }^{2}$ and Gunwoo Lee $\mathbb{D}^{2}$ \\ ${ }^{1}$ Department of Smart City Research, Seoul Institute of Technology, Maebongsan-ro 37, Mapo-gu, Seoul, 03909, Republic of Korea \\ ${ }^{2}$ Department of Transportation and Logistics Engineering, ERICA Campus-Hanyang University, 55 Hanyangdaehak-ro, \\ Sangnok-gu, Ansan, Gyeonggi-do 15588, Republic of Korea \\ Correspondence should be addressed to Cheol Oh; cheolo@hanyang.ac.kr
}

Received 13 July 2020; Revised 2 December 2020; Accepted 2 January 2021; Published 28 January 2021

Academic Editor: Hesham Rakha

Copyright (c) 2021 Seolyoung Lee et al. This is an open access article distributed under the Creative Commons Attribution License, which permits unrestricted use, distribution, and reproduction in any medium, provided the original work is properly cited.

Vehicle platooning service through wireless communication and automated driving technology has become a reality. Vehicle platooning means that several vehicles travel like a train on the road with a minimum safety distance, which leads to the enhancement of safety, mobility, and energy savings. This study proposed a framework for exploring traffic mobility and safety performance due to the market penetration rate (MPR) of truck platoons based on microscopic traffic simulations. A platoon formation algorithm was developed and run on the VISSIM platform to simulate automated truck maneuvering. As a result of the mobility analysis, it was found that the difference in network mobility performance was not significant up to MPR 80\%. Regarding the mobility performance of the truck-designated lane, it was found that the average speed was lower than in other lanes. In the truck-designated lane of the onramp section, the average speed was identified to be approximately $33 \%$ lower. From the viewpoint of network safety, increasing the MPR of the truck platoon has a positive effect on longitudinal safety but has a negative effect on lateral safety. The safety analysis of the truck-designated lane indicated that the speed difference by lane of MPR $100 \%$ is 2.5 times higher than that of MPR $0 \%$. This study is meaningful in that it explores traffic flow performance on mobility and safety in the process of platoon formation. The outcomes of this study are expected to be utilized as fundamentals to support the novel traffic operation strategy in platooning environments.

\section{Introduction}

The development of automated vehicles technology is expected to transform the roads now used as movement spaces into productive spaces, which will lead to unprecedented changes in human life. One of the services using automated vehicles is vehicle platooning, which means that several vehicles travel together at a minimum safety distance through V2X (vehicle-to-everything) communication technology. Platooning services will be applied primarily to trucks because of their characteristics. In the case of trucks, accidents caused by human factors such as drowsiness are frequent and are likely to be large. The typical expected effects of platooning include enhanced safety, fuel saving, and environmental improvement, along with an economic effect. In terms of safety, autonomous driving and V2X technology allow vehicles in the platoon to avoid traffic accidents by quickly responding to unexpected situations [1-4]. In addition, since several vehicles travel at short intervals, the air resistance is reduced, and fuel is saved, thereby enhancing the competitiveness of the truck [5-7]. Finally, it is expected that the platooning can shorten the travel time by increasing the road capacity, and the trucks are expected to contribute to the reduction in the labor force by reducing the workload of the truck driver through the platooning because trucks are characterized by frequent overnight or long-distance operations [8-11].

The technology for automated vehicles and wireless communications, infrastructure, the legal system, and traffic operations and management plans should be developed before platooning can be commercialized. According to the EU truck platooning roadmap, the trucks, except for the leader in the platoon, are expected to reach the SAE level 4, which means they are capable of autonomous driving without a driver by 
2025 [12]. In addition, the V2X communication required to realize such technology is rapidly advancing, and 5th generation communication technology $(5 \mathrm{G})$ is being commercialized. This developing technology will provide information to the automated vehicle to enable a prompt response when an unexpected situation occurs. It will be possible to provide realtime information using big data through the cooperative intelligent transport systems (C-ITS) infrastructure [13]. For truck platooning, legal standards and operational strategies related to automated vehicles should be established. Although some studies have been conducted on automated vehicle-only lanes [14], the automated vehicle must be mainstream to validate the operation of an automated vehicle-designated lane. However, early in its introduction, the truck platoon will be driven on existing truck restricted lanes. Therefore, it is necessary to prepare for situations in which there is no platoononly lane. In addition, platooning can have a negative effect on the surrounding general vehicles, and the performance of the entire traffic flow can be lowered by the interactions between the vehicle platoons and the general vehicles. In the platooning environment, the possibility of a general vehicle lane change failure increases, and the driver's psychological anxiety can be increased in the process of switching control from manual driving to automated driving $[11,15]$. In preparation for such mixed traffic conditions, it is necessary to develop an effective traffic management strategy to optimize the performance of the road traffic system by understanding the interactions between the vehicle platoons and general vehicles.

There have been many studies using driving simulation and microtraffic simulation to evaluate the effects of automated vehicles and platooning in previous studies [16-18]. Existing research on platooning focuses on the safety analysis of the platoon and the effect of a platoon dedicated lane $[14,19]$. However, under the present legal system, trucks are allowed to run on designated lanes, and there have been no studies on the new operational strategy view of the situation in which there is no platoon dedicated lane. The mobility performance and merging characteristics were evaluated in the previous studies for identifying the effectiveness of truck platooning $[20,21]$. However, it is necessary to evaluate the traffic safety of mixed traffic flow by identifying the interactions between truck platoons and general vehicles. Therefore, it is necessary to develop a management strategy for the platooning to improve the mobility and safety of the traffic flow in terms of traffic management. The purpose of this study is to present the platoon formation algorithm and apply it to a simulation to identify the effect of the truck platoon on the freeway onramp area according to the market penetration rate (MPR) of the truck platoon. The proposed algorithm consists of three components: the truck sequencing in the platoon, the determination of platoon completion, and a speed control algorithm. To discover the effect of the truck platoon on traffic flow, we implemented a truck platoon in the COM interface environment of VISSIM, a microtraffic simulation program. The platoon MPR of trucks was used as an analysis scenario component. The effects of truck platooning on the on-ramp were identified in terms of traffic mobility and safety according to the MPR.
The remainder of this paper is organized as follows. Section 2 presents the proposed simulation methodology including an overall analysis framework incorporating the proposed platoon formation algorithm. Section 3 presents the analysis results and discussions for traffic mobility and safety. Finally, Section 4 presents the conclusion with a summary of this study and future research directions based on the limitations identified while conducting this study.

\section{Methodology}

2.1. Overall Framework. An overall framework consisting of three steps is presented in Figure 1. Step 1 includes the proposed platoon formation algorithm developed in this study. Step 2 is the simulation experiments and consists of the section for setting scenarios and networks and the section for conducting simulations using VISSIM. In this study, the rate of platooning among trucks is defined as the MPR, and the MPR is a scenario component. The COM interface collects the trajectory data of each individual vehicle at each time step and controls the speed of the individual trucks in the platoon according to the platoon formation algorithm. Step 3 identifies the mobility and safety performance from the aspect of the overall performance of the network and the performance on the truck platoon designation lane using individual vehicle trajectory data derived from the simulation.

\subsection{Development of the Platoon Formation Algorithm.} Three important parameters including platoon size, intraplatoon spacing, and interplatoon spacing need to be determined for the truck platooning. The platoon size represents the number of trucks in the platoon, and the interplatoon spacing is the spacing between the trucks in the platoon. On the other hand, the intraplatoon spacing means the spacing between platoons. These parameters can support decision making for establishing platoon operation strategies in different traffic conditions. The platooning algorithms in previous studies attempted to maintain platoons based on intelligent driver models (IDM) [14, 17]. Unlike existing studies, the proposed platoon formation algorithm in this study is an algorithm that is designed to form platoons based on the aforementioned platoon formation parameters for the purpose of analyzing the interaction between the platoons and the general vehicles more effectively. In addition, a nice feature of the proposed algorithm is the implementation of the operating situation of autonomous emergency braking systems (AEBS) by changes in acceleration patterns.

The platoon formation algorithm is shown in Figure 2, and the parameters associated with the platoon formation algorithm are defined in Table 1. The proposed algorithm consists of three components, which are the truck sequencing in the platoon (Part 1), the determination of platoon completion (Part 2), and the speed control algorithm (Part 3). In Part 1 and Part 3, the algorithm proceeds on an individual vehicle basis in the platoon, and in Part 2, the algorithm proceeds on a platoon basis. The truck sequencing algorithm determines the order of the subject 


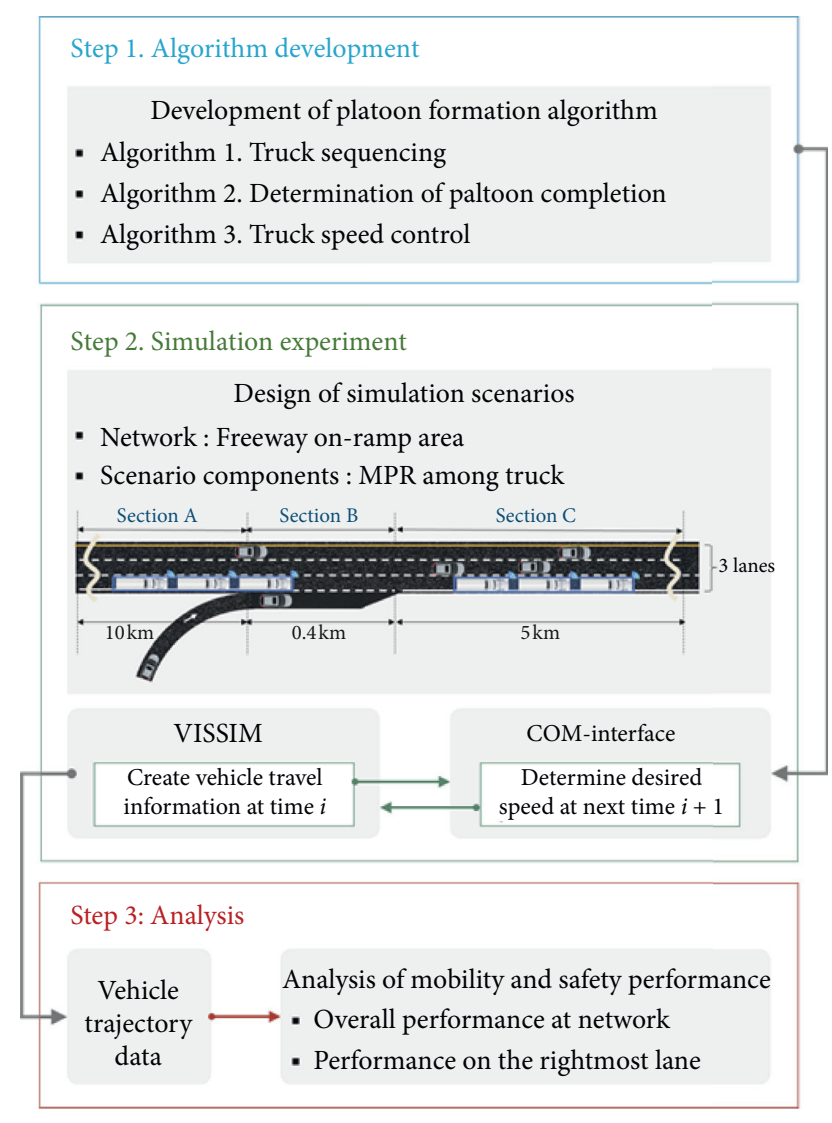

Figure 1: Overall framework.

vehicles in the platoon and assigns the sequence number of each truck, as shown in Part 1 . Here, a vehicle designated as the platoon leader $\left(\operatorname{Lead}_{\mathrm{PL}}\right)$ does not give up the role of the platoon leader unless an exceptional case occurs, which means that a truck capable of forming the platoon enters in front of the leader $\left(\operatorname{Lead}_{\mathrm{PL}}\right)$ when the platoon size does not exceed a predefined maximum threshold $\left(\operatorname{Max}_{\mathrm{PL}}\right)$. The sequence number $\left(\mathrm{Ord}_{\mathrm{PL}}\right)$ of the new platoon leader is designated as 1 , and the sequence number of the following platoon members is set to the next sequence number from the truck in the front. The completion of the platoon defined in this study is a case where the platoon size reaches a predefined maximum value $\left(\mathrm{Max}_{\mathrm{PL}}\right)$. It is assumed that a new truck cannot enter the completed platoon. The platoon size that is currently being formed before reaching the maximum platoon size is indicated by $\mathrm{PL}_{\mathrm{Con}}$ in Part 1 . It is expected that a traffic operations agency can implement a traffic management strategy that controls the platoon formation parameters while monitoring the driving conditions of the platoon using the sequencing information.

The second algorithm illustrated in Part 2 is to determine whether the platoon formation is completed or not. In the case of the incomplete platoon, it provides information on the platoon size being formed by all trucks in the platoon $\left(\mathrm{PL}_{\mathrm{Con}}\right)$. On the other hand, if the platoon is completed, all trucks in the platoon share the information that the platoon has been completed $\left(\mathrm{PL}_{\mathrm{Com}}\right)$. The information obtained from the proposed determination algorithm for platoon completion is used in the truck sequencing algorithm in the next simulation time step.

The last component is a speed control algorithm, as shown in Part 3, which manages the desired speed and spacing of the trucks in the platoon. First, the algorithm identifies the sequence number of the subject truck and checks the required speed and spacing accordingly. For example, if there is no preceding vehicle, the subject vehicle travels at the platoon-keeping speed $\left(V_{\mathrm{PL}}\right)$, and the leader and platoon members in which the preceding vehicle exists attempt to travel while maintaining the interplatoon spacing and the intraplatoon spacing. Under these circumstances, the acceleration of the subject vehicle is adjusted to achieve the desired speed and spacing. Then, the vehicle maneuvering is determined by two driving modes including the comfort driving mode and the emergency braking mode based on the time to collision (TTC). The acceleration rates in this application adopted values proposed in the literature $[16,22]$. The acceleration at which AEBS operated in this study is derived from equations (1)-(3) based on the threshold recommended by the literature [22]. In the comfort mode, where the inverse TTC is less than 0.68 seconds, equation (1) is applied when the acceleration is required. On the other hand, when deceleration is required, the acceleration is determined by equation (2). When the inverse TTC is 0.68 seconds or longer, the AEBS is activated in this study because it is expected that a dangerous situation to require rapid deceleration would occur. Equation (3) presents an acceleration to be used for activating the AEBS, which is $-6\left(\mathrm{~m} / \mathrm{s}^{2}\right)$. The speed at the next time step according to the acceleration derived from equations (1)-(3) is obtained by equation (4). Additionally, a newly estimated speed does not exceed the speed limit and the minimum speed. To achieve the desired speed and spacing, the obtained speed is used as the speed of the next simulation time step. The VISSIM COM interface is used for updating the speed at every second.

Comfort mode to require acceleration:

$\operatorname{Acc}\left(\mathrm{m} / \mathrm{s}^{2}\right)= \begin{cases}1.5, & \text { if } V \leq 40(\mathrm{~km} / \mathrm{h}), \\ -\frac{1}{60} V+\frac{10}{3}, & \text { if } 40(\mathrm{~km} / \mathrm{h})<V \leq 70(\mathrm{~km} / \mathrm{h}), \\ 1, & \text { if } V>70(\mathrm{~km} / \mathrm{h}) .\end{cases}$

Comfort mode to require deceleration:

$\operatorname{Acc}\left(\mathrm{m} / \mathrm{s}^{2}\right)= \begin{cases}-2, & \text { if } V \leq 40(\mathrm{~km} / \mathrm{h}), \\ \frac{1}{30} V-\frac{10}{3}, & \text { if } 40(\mathrm{~km} / \mathrm{h})<V \leq 70(\mathrm{~km} / \mathrm{h}), \\ -1, & \text { if } V>70(\mathrm{~km} / \mathrm{h}) .\end{cases}$

AEBS mode to require rapid deceleration: 
Part 1. Truck sequencing algorithm

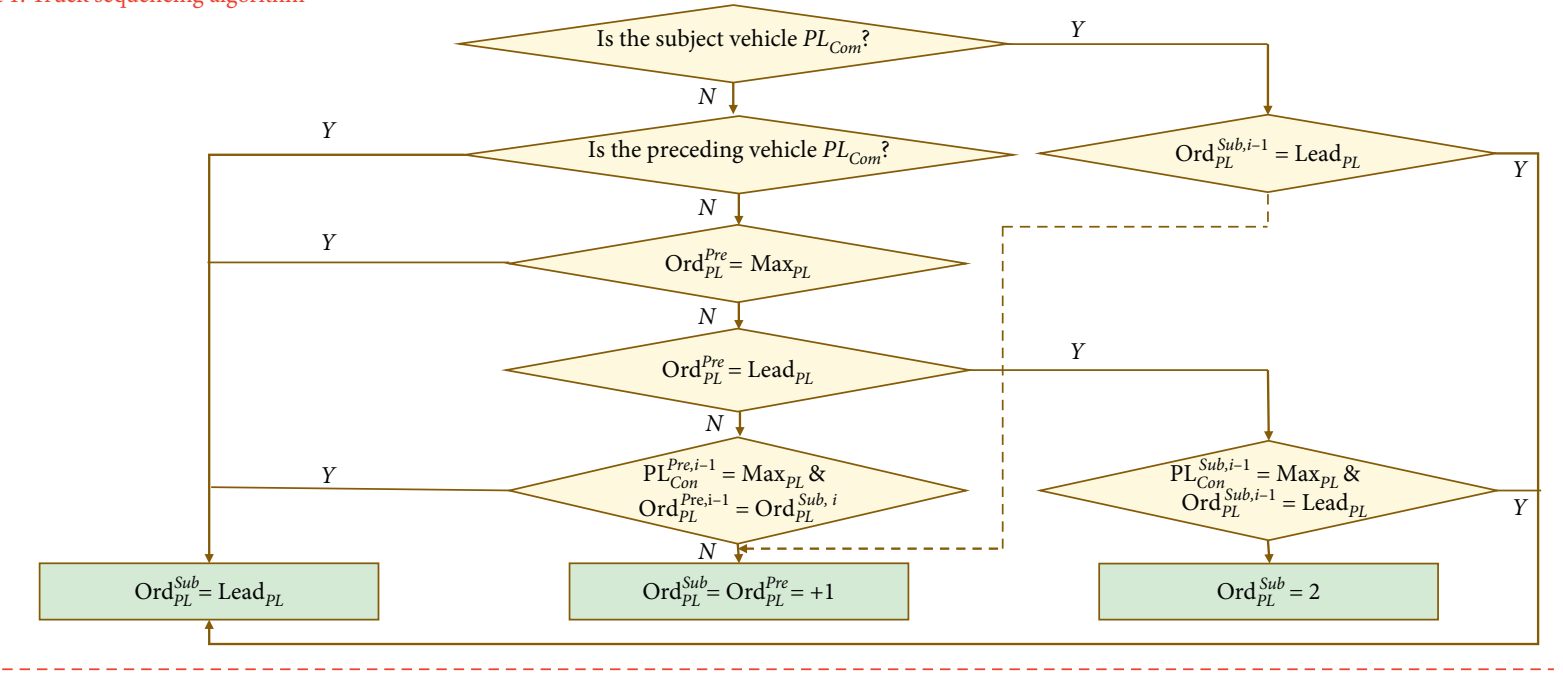

Part 2. Determination of platoon completion algorthim

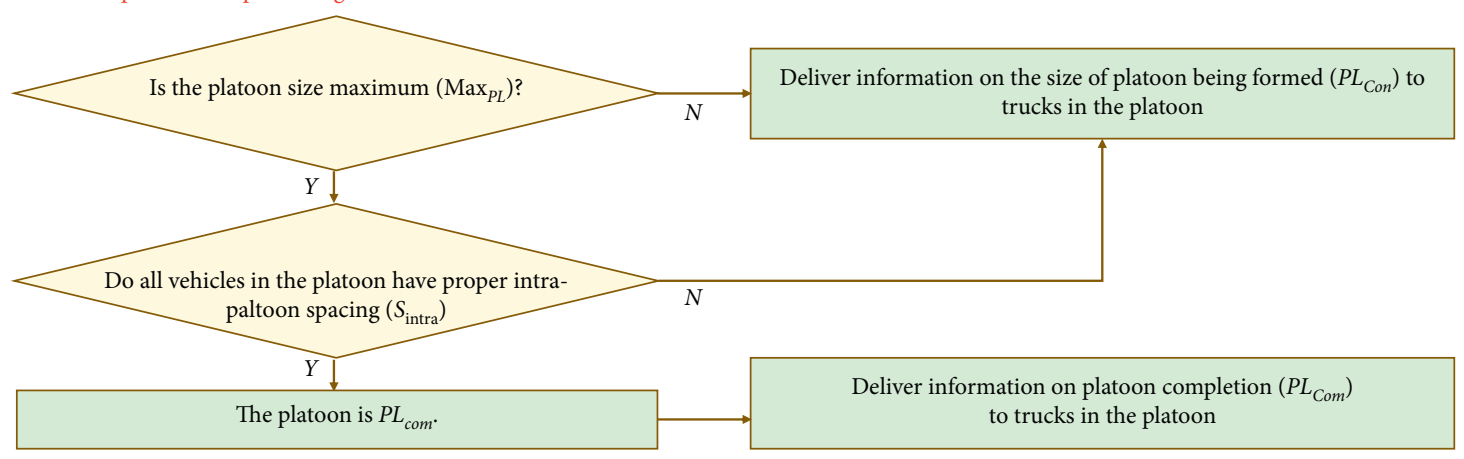

Part 3. Truck speed control algorithm

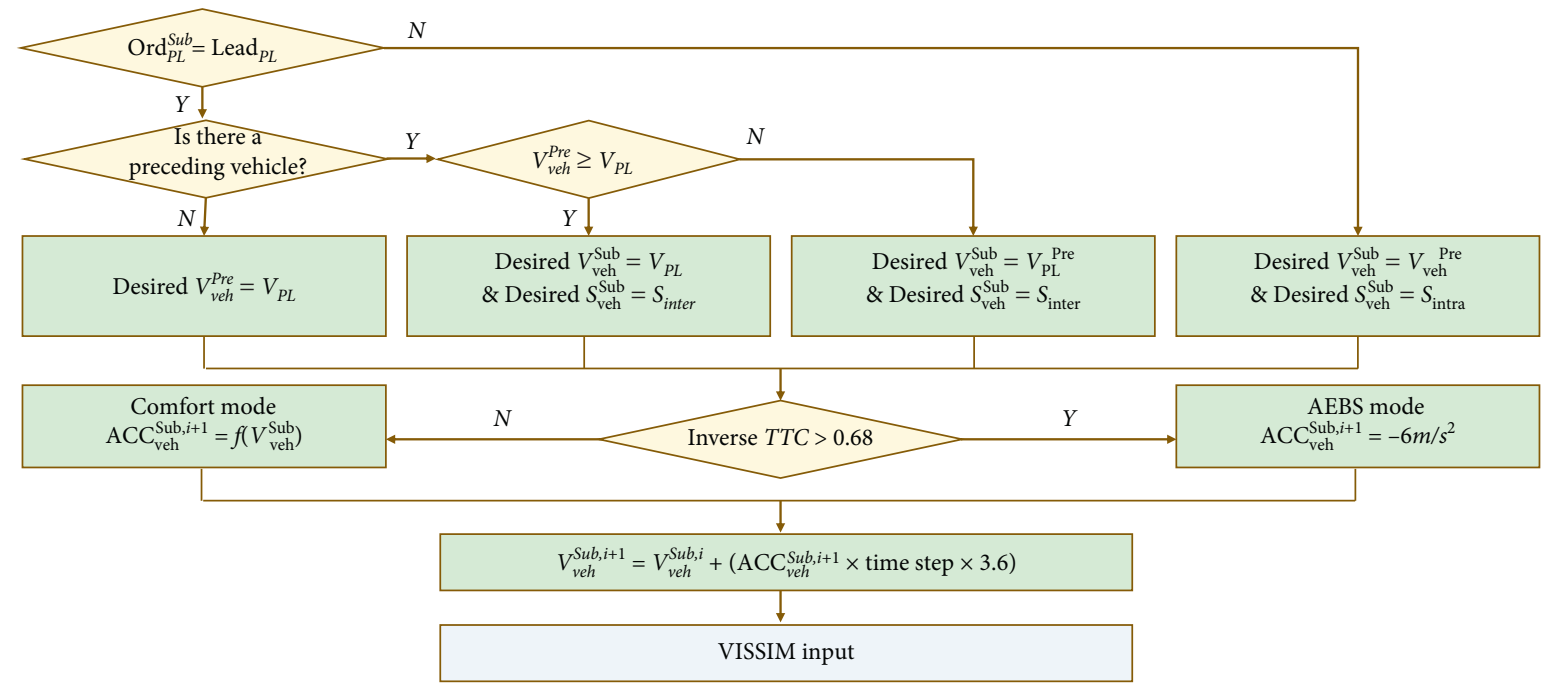

FIgURE 2: Proposed platoon formation algorithm. 
TABLE 1: Definition of terms used in platoon formation algorithms.

\begin{tabular}{ccc}
\hline Category & Notation & Description \\
\hline \multirow{4}{*}{ Platoon } & $\mathrm{PL}_{\mathrm{Com}}$ & Completed platoon \\
& $\mathrm{PL}_{\mathrm{Con}}$ & The size of the platoon being formed \\
& $\mathrm{Max}_{\mathrm{PL}}$ & Maximum platoon size \\
& $\mathrm{Ord}_{\mathrm{PL}}$ & Vehicle order \\
& $\mathrm{Lead}_{\mathrm{PL}}$ & Platoon leader/Leading truck \\
& $V_{\mathrm{PL}}$ & Platoon-keeping speed \\
& $S_{\text {Intra }}$ & Intraplatoon spacing \\
& $S_{\text {Inter }}$ & Interplatoon spacing \\
\hline \multirow{4}{*}{ Vehicle } & $V_{\text {veh }}$ & Vehicle speed \\
& $S_{\text {veh }}$ & Vehicle spacing \\
& Acc & Acceleration/Deceleration \\
\hline \multirow{3}{*}{ Superscript } & Sub & Subject vehicle \\
& Pre & Preceding vehicle \\
& $i$ & Time step \\
\hline
\end{tabular}

$$
\begin{aligned}
\operatorname{Acc}\left(\mathrm{m} / \mathrm{s}^{2}\right) & =-6, \\
V^{i+1} & =V^{i}+\left(\operatorname{Acc}^{i+1} \times \text { time step } \times 3.6\right) .
\end{aligned}
$$

2.3. Analysis Environments. The simulation experiments were conducted to evaluate the impacts of freeway truck platooning on traffic mobility and safety by applying the proposed platoon formation algorithm through the VISSIM software. The range of spatial analysis was selected as the freeway on-ramp section. From the perspective of freeway traffic safety, the on-ramp area is of keen interest because frequent merging situations based on the interaction between platoons and general entering vehicles lead to a high crash potential. The other consideration is that, according to the Korean Road Traffic Act, the trucks must travel in the rightmost lane on a three-lane freeway segment, which is the designated lane for trucks in Korea. Existing studies evaluated the effectiveness when the leftmost lane was used for the high-occupancy lane (HOV) or dedicated lane [21, 23]. On the other hand, this study conducted simulation experiments based on the current Korean traffic regulation that trucks should travel in the designated rightmost lane on freeways. Thus, the analysis network is set up as a 3-lane $15.4 \mathrm{~km}$ virtual freeway with an on-ramp, and the network structure is shown in Figure 1. Then, the truck platooning was restricted to only the rightmost lane. The section from the network starting point up to $10 \mathrm{~km}$ is section $\mathrm{A}$, with no change in geometry. Section B is a $0.4 \mathrm{~km}$ on-ramp located next to section A. Finally, section $C$ is the $5 \mathrm{~km}$ main line after the on-ramp.

The demand volume of the mainline freeway is $3,500 \mathrm{veh} / \mathrm{h}$, which corresponds to traffic conditions representing a level of service (LOS) C based on the Korean Highway Capacity Manual [24]. This is because the design level of service of the freeway is the C-D level. In addition, the Korean Policy for Road Geometry Designs was used to determine the geometric conditions to install a one-lane on-ramp in the simulation network [25].
It is assumed that all the entering vehicles are passenger cars on a one-lane on-ramp with an initial speed of $60 \mathrm{~km} /$ h. An on-ramp traffic volume of $500 \mathrm{veh} / \mathrm{h}$ was used in this application. The proportions of the vehicle types were determined by the actual observations from the Gyeongbu freeway. The proportions of passenger cars, trucks, and buses are $79 \%, 17 \%$, and $4 \%$, respectively. The speed limit of trucks is $90 \mathrm{~km} / \mathrm{h}$, and the minimum speed is $50 \mathrm{~km} / \mathrm{h}$. The platoon-keeping speed is $80 \mathrm{~km} / \mathrm{h}$ based on the assumption that the autonomous truck capable of forming platoons must follow the traffic regulation. This is because the trucks cannot accelerate when the platoon speed is equal to the speed limit.

Traffic mobility and safety analysis were conducted with an interplatoon spacing of $5 \mathrm{~m}$, an intraplatoon spacing of $50 \mathrm{~m}$, and platoon sizes of $2,4,6,8$, and 10 , which were determined based on existing studies dealing with field and simulation implementation [26-28]. To evaluate the impact of an increased number of trucks capable of platooning, the MPR was increased from $0 \%$ to $100 \%$ at $20 \%$ intervals. As a result, a total of 6 scenarios were determined by considering MPRs. To increase the reliability of the simulation results, different random seeds were applied, and 5 simulation runs were conducted for each scenario. Then, the average of the results of the five runs was presented. The simulation analysis time was $2,000-\mathrm{sec}$, and a warm-up time period of $600-\mathrm{sec}$ was provided to ensure an even distribution of the vehicles across the network.

2.4. Simulation Calibrations. The simulation calibration was conducted to make simulation experiments more realistic based on the comparison of actual traffic data and simulation data. Traffic speed data collected from the vehicle detection systems installed on the Shingal JC-Kiheung IC on the Gyeongbu freeway were used for the calibration in this study. This section of the freeway has been equipped with connected vehicle systems infrastructure for a truck platooning pilot study. "U-statistic" presented in equation (5) was used to determine the acceptability of simulation results. In general, u-statistic close to 0 represents that actual and simulated data are similar. In case the $\mathrm{u}$-statistic is less than 0.1 , calibration results can be accepted [29]. The result of deriving the average of $U$-statistic obtained by repeating simulations 5 times is presented in Table 2. It can be said that the calibration result is acceptable because $U$-statistics less than 0.1 are achieved.

$$
U=\frac{\sqrt{\sum_{i=1}^{N}\left(V_{i}-\widehat{V}_{i}\right)^{2}}}{\sqrt{\sum_{i=1}^{N} V_{i}^{2}}+\sqrt{\sum_{i=1}^{N} \widehat{V}_{i}^{2}}},
$$

where $V_{i}$ is the actual speed $(\mathrm{km} / \mathrm{h})$ and $\widehat{V}_{i}$ is the simulation speed $(\mathrm{km} / \mathrm{h})$.

In addition to the simulation calibration aforementioned, this study adjusted the Wiedemann 99 model parameters of VISSIM using the recommended values presented in the literature [30] to simulate the 
TABle 2: Comparison of U-values between field and simulation data.

\begin{tabular}{lcccccc}
\hline & \multicolumn{7}{c}{ Iterations } & Average \\
& 1 & 2 & 3 & 4 & 5 & \\
\hline Detector 1 & 0.019 & 0.018 & 0.019 & 0.019 & 0.018 & 0.019 \\
Detector 2 & 0.064 & 0.063 & 0.064 & 0.064 & 0.063 & 0.064 \\
Detector 3 & 0.026 & 0.024 & 0.025 & 0.026 & 0.025 & 0.025 \\
Detector 4 & 0.066 & 0.064 & 0.066 & 0.066 & 0.066 & 0.066 \\
Detector 5 & 0.057 & 0.055 & 0.058 & 0.058 & 0.057 & 0.057 \\
\hline
\end{tabular}

longitudinal and lateral behavior of the truck platoon. Adjusted parameters are presented in Table 3. Parameter values corresponding to the autonomous driving level 4 defined by the American Society of Automotive Engineers (SAE) were adopted. In our simulation experiment, a truck platoon travels based on the proposed platoon formation algorithm. On the other hand, parameters presented in Table 3 are adopted for simulating the maneuvering of individual autonomous trucks to which the platooning algorithm is not applied.

\section{Analysis}

\subsection{Mobility Performance}

3.1.1. Overall Mobility Performance of the Network. The mobility analysis of the network was conducted based on time and space using the average speed indicator. The simulation network for analyzing spatiotemporal patterns of mobility consists of section B with on-ramp, $2 \mathrm{~km}$ of section $A$, and $1 \mathrm{~km}$ of section $C$, which are upstream and downstream sections around section $\mathrm{B}$, respectively. For the
$3.4 \mathrm{~km}$ analysis space, we constructed 34 space cells in a $100 \mathrm{~m}$ unit and 40 time cells in $50 \mathrm{sec}$ for a 2,000-sec analysis time. A total of 1,360 time-space cells were used for the spatiotemporal analysis. The average speed $(V)$ of each cell was quantified and classified according to the categorization of congestion levels $(k)$ on Korean freeways such as congestion-free, light congestion, and heavy congestion as presented in equation (6). If the average speed of a cell is more than $80 \mathrm{~km} / \mathrm{h}$, it is classified as congestion-free. Light congestion is classified as $40 \mathrm{~km} / \mathrm{h}-80 \mathrm{~km} / \mathrm{h}$ and heavy congestion is less than $40 \mathrm{~km} / \mathrm{h}$. After counting the number of classified congestion levels, the number of time-space cells by congestion level against the total number of cells was quantified and presented as in equation (7). Table 4 shows the results of analyzing the rate of congestion (ROC) by congestion level according to MPR. As a result, heavy congestion did not occur in all MPRs, and the mobility performance difference between the MPR $0-80 \%$ was insignificant. However, the scenario in which light congestion cells occupy the largest percentage is MPR $100 \%$, and the ROC in this scenario is identified as 13\%. For the MPR 100\% scenarios with the lowest mobility performance, the results of the average speed according to spatiotemporal analysis were additionally presented. As a result of the analysis, it was found that the speed decreases slightly in section B, which affects the speed of the upstream section $\mathrm{A}$ as time goes by. The reason for the decrease in mobility performance is that the vehicles entering from the on-ramp change their path into or between platoons for the lane change. In particular, it is believed that the performance is poor at $100 \% \mathrm{MPR}$ because trucks travel at a lower platoon-keeping speed compared to the speed of general vehicles.

$$
\begin{gathered}
\text { Categorization of congestion level }= \begin{cases}\text { Congestion }- \text { free }(k=1), & 80(\mathrm{~km} / \mathrm{h}) \leq V, \\
\text { Light congestion }(k=2), & 40(\mathrm{~km} / \mathrm{h}) \leq V<80(\mathrm{~km} / \mathrm{h}), \\
\text { Heavy congestion }(k=3), & V<40(\mathrm{~km} / \mathrm{h}),\end{cases} \\
\operatorname{ROC}_{k}=\frac{\text { number of cells for congestion level } k}{\text { total number of cells in time }- \text { space diagram }} * 100(\%),
\end{gathered}
$$

where $k$ is the level of congestion, $V$ is the average speed, $R O C$ is the rate of congestion, and $m$ is the count.

3.1.2. Mobility Performance in the Rightmost Lane. The general vehicles entering from an on-ramp need to make a compulsory lane change to enter the main line. Traffic operation management in the on-ramp is necessary so that general vehicles can change lanes more safely. In the analysis network, the rightmost lane is the truck-designated lane, and the analysis of mobility performance is performed for the rightmost lane. The average speed results for the lanes selected as mobility indicators are presented in Figure 3. As a result of the analysis, the average speed of all lanes in section $B$ is lower than that in sections A and C. Furthermore, the average speed of the rightmost lane in $\mathrm{A}$ and $\mathrm{C}$ sections is approximately $10 \%$ lower than that of the other lanes, while it is approximately 33\% lower in B section. Because trucks drive at lower speeds than passenger cars, the average speed of the rightmost lane is seen as low. In section B especially, the speed of the rightmost lane seems to be lower because of the situations in which the truck that is driving in the main line decelerates due to the entering vehicle. Therefore, it is necessary to establish a strategy to improve the mobility of the truck-designated lane in the on-ramp through the speed control of the truck platoon. 
TABLE 3: Platooning implementation parameters [30].

\begin{tabular}{|c|c|c|c|}
\hline Division & Code & VISSIM default & Automated vehicle (SAE level 4) \\
\hline Spacing & $\begin{array}{l}\text { Standstill distance }(\mathrm{CC} 0) \\
\text { Headway time }(\mathrm{CC} 1) \\
\text { Following variation }(\mathrm{CC} 2)\end{array}$ & $\begin{array}{l}1.50 \mathrm{~m} \\
0.90 \mathrm{~s} \\
4.00 \mathrm{~m}\end{array}$ & $\begin{array}{l}0.5 \mathrm{~m} \\
0.6 \mathrm{~s} \\
0 \mathrm{~m}\end{array}$ \\
\hline Speed & $\begin{array}{l}\text { Threshold for entering following (CC3) } \\
\text { Negative following threshold (CC4) } \\
\text { Positive following threshold (CC5) }\end{array}$ & $\begin{array}{c}-8.00 \mathrm{~s} \\
0.41 \mathrm{~km} / \mathrm{h} \\
0.41 \mathrm{~km} / \mathrm{h} \\
\end{array}$ & $\begin{array}{c}0 \mathrm{~s} \\
0 \mathrm{~km} / \mathrm{h} \\
0 \mathrm{~km} / \mathrm{h}\end{array}$ \\
\hline Acceleration & $\begin{array}{c}\text { Speed dependency of oscillation (CC6) } \\
\text { Oscillation acceleration (CC7) } \\
\text { Standstill acceleration (CC8) }\end{array}$ & $\begin{array}{c}11.44 \\
0.25 \mathrm{~m} / \mathrm{s}^{2} \\
3.50 \mathrm{~m} / \mathrm{s}^{2}\end{array}$ & $\begin{array}{c}0 \\
0.4 \mathrm{~m} / \mathrm{s}^{2} \\
3.8 \mathrm{~m} / \mathrm{s}^{2}\end{array}$ \\
\hline Lane change & $\begin{array}{c}\text { Min. headway } \\
\text { Safety distance reduction factor }\end{array}$ & $\begin{array}{c}0.5 \mathrm{~m} \\
0.6\end{array}$ & $\begin{array}{c}0.2 \mathrm{~m} \\
0.3\end{array}$ \\
\hline Priority rule & $\begin{array}{l}\text { Min. time gap } \\
\text { Min. headway }\end{array}$ & $\begin{array}{c}3 \mathrm{~s} \\
5 \mathrm{~m}\end{array}$ & $\begin{array}{c}2.4 \mathrm{~s} \\
3.5 \mathrm{~m}\end{array}$ \\
\hline Others & $\begin{array}{c}\text { Acceleration with } 80 \mathrm{~km} / \mathrm{h} \text { (CC9) } \\
\text { Look-ahead distance observed vehicles } \\
\text { Smooth closeup behavior }\end{array}$ & $\begin{array}{l}1.50 \mathrm{~m} / \mathrm{s}^{2} \\
2 \text { vehicles } \\
\text { No }\end{array}$ & $\begin{array}{l}1.8 \mathrm{~m} / \mathrm{s}^{2} \\
10 \text { vehicles } \\
\text { Yes }\end{array}$ \\
\hline
\end{tabular}

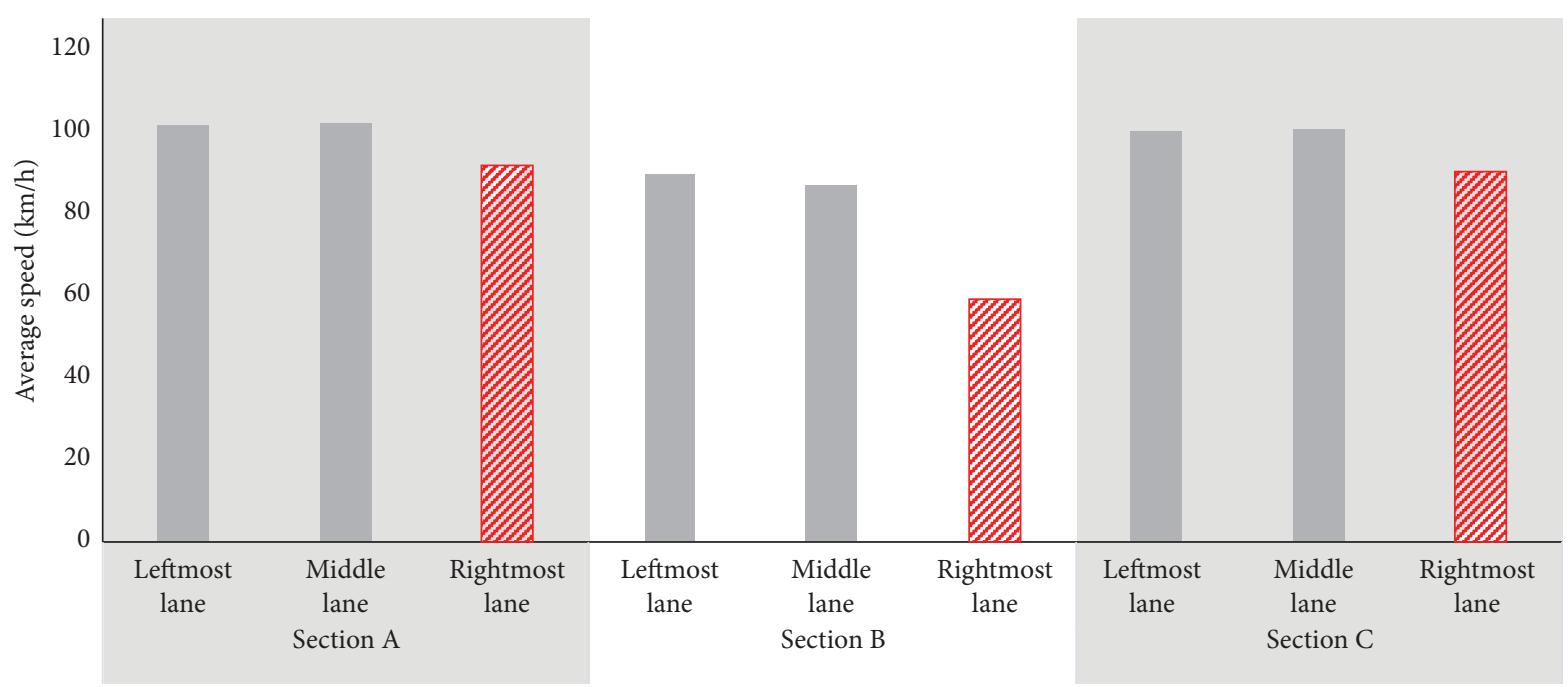

FIgURE 3: Comparison of speed by lanes for each section.

\subsection{Safety Performance}

3.2.1. Overall Safety Performance of the Network. This study suggests the characteristics of longitudinal and lateral conflicts by time and space according to the MPR. First, rear-end conflicts were selected as a longitudinal safety indicator. The rear-end conflicts can be identified using the safety distance (FD) and the follow distance (SD) derived from the simulation. The situation in which the SD is greater than the FD is defined as a conflict and represents a shorter distance than the minimum safe distance between a lead and the following vehicle [31]. A method of determining a conflict based on the analysis of SD and FD is expressed in equation (8). The number of conflicts $(C)$ is quantified in the same time-space interval $(t, s)$ as the mobility analysis. Equation (9) represents the number of conflicts for all vehicles $(N)$ in a cell. A risk-free condition is defined for the numerical comparison of safety according to the MPR, and the risk-free condition means a situation in which there is no conflict. In this study, the case in which the number of conflicts in a single cell is 0 was defined as a risk-free cell (RFC), which is presented in equation (10). The ratio of RFCs to a total number of cells is defined as the rate of risk-free conditions (RORF). Figure 4(a) shows the RORF for each MPR derived using equation (11).

$$
\begin{gathered}
\text { conflict determination }= \begin{cases}\text { conflict, } & \mathrm{SD}>\mathrm{FD}, \\
\text { not conflict, } & \mathrm{SD} \leq \mathrm{FD},\end{cases} \\
C_{(t, s)}=\sum_{j=1}^{N} m\left(\mathrm{SD}_{j}>\mathrm{FD}_{j}\right), \\
\mathrm{RFC}=\sum_{t=1}^{T} \sum_{s=1}^{S} m\left(C_{(t, s)}=0\right), \\
\mathrm{RORF}=\frac{\mathrm{RFC}}{\text { total number of cells }} * 100(\%),
\end{gathered}
$$


TABLE 4: Rate of congestion level by MPR.

\begin{tabular}{|c|c|c|c|c|c|c|c|}
\hline \multirow{2}{*}{\multicolumn{2}{|c|}{ Freeway congestion level }} & \multicolumn{6}{|c|}{ MPR } \\
\hline & & $0 \%$ & $20 \%$ & $40 \%$ & $60 \%$ & $80 \%$ & $100 \%$ \\
\hline Congestion-free & Above $80 \mathrm{~km} / \mathrm{h}$ & $97 \%$ & $96 \%$ & $96 \%$ & $95 \%$ & $96 \%$ & $87 \%$ \\
\hline Light congestion & $40 \mathrm{~km} / \mathrm{h}-80 \mathrm{~km} / \mathrm{h}$ & $3 \%$ & $4 \%$ & $4 \%$ & $5 \%$ & $4 \%$ & $13 \%$ \\
\hline Heavy congestion & Under $40 \mathrm{~km} / \mathrm{h}$ & $0 \%$ & $0 \%$ & $0 \%$ & $0 \%$ & $0 \%$ & $0 \%$ \\
\hline
\end{tabular}

Spatiotemporal diagram of speed (MPR 100\%)

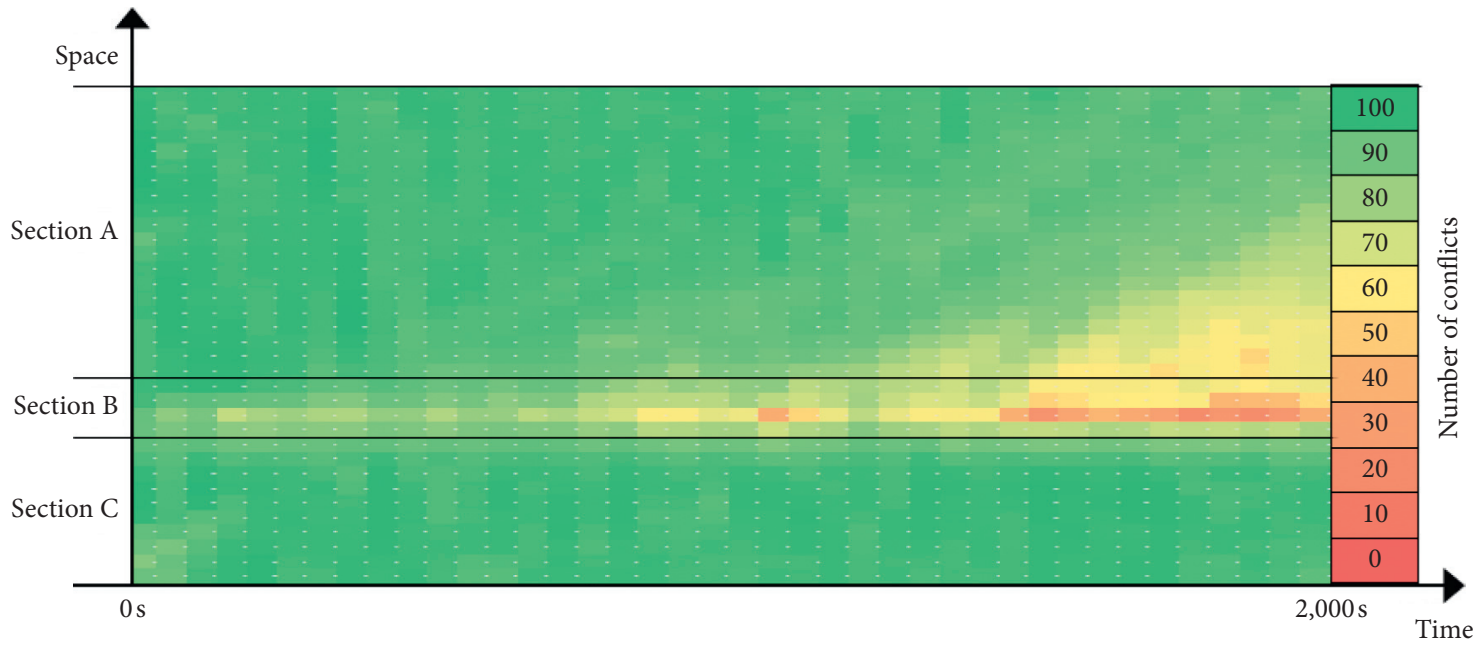

where SD is the safety distance, FD is the follow distance, $C$ is the number of conflicts, $m$ is the count, $t$ is the time cell, $s$ is the space cell, $j$ is the number of vehicles, $N$ is the total number of vehicles, $T$ is the number of time cells, $S$ is the number of space cells, RFC is the risk-free cells, and RORF is the rate of risk-free conditions.

The results of the RORF analysis indicated that safety increases with increasing MPR. In particular, the RORF of MPR $100 \%$ was $51 \%$, and it was observed that no rear-end conflicts occurred in more than half of the cells. The spatiotemporal analysis was performed for MPR 0\% with the lowest RORF and MPR 100\% with the highest RORF. The results of the spatiotemporal diagram analysis of the number of conflicts are shown in Figure 4(b). In both scenarios, it was found that the rear-end conflicts frequency was high at the on-ramp. At the MPR $0 \%$, which is the lowest safety scenario, the number of rear-end conflicts in the B section was estimated to be approximately 1.42 times higher than that in the A and C sections. In the case of MPR 100\%, which showed the highest safety, it was found that the average number of rear-end conflicts in all sections was approximately 5 conflicts lower. It has been identified that the increase in truck platoon positively affects the increase in longitudinal safety of all sections.

The lane change conflict was defined and used as an indicator of lateral safety. In this study, we used information such as the speed and coordinates of individual vehicles derived from simulations to identify the lane change conflict. According to the SSAM report, conflict is classified as a lane change conflict when the starting point and ending point of the conflict occur in different lanes and the collision angle between the two vehicles is $-30^{\circ}$ to $-85^{\circ}$ or $30^{\circ}$ to $85^{\circ}$ [32].
Therefore, the TTC was calculated according to equation (12) for vehicles in the lane change conflict range. The conflict threshold using the TTC proposed in the previous study is 1.5 seconds, and the method of determining the conflict in equation (13) is described [33]. The formula for calculating the number of conflicts by car is shown in equation (14) and the RORF is calculated in the same way as the number of rear-end conflicts.

$$
\begin{aligned}
\text { TTC } & =\frac{\sqrt{x^{2}+y^{2}}}{V_{s}-V_{t}}, \quad \forall V_{s}>V_{t}, \\
\text { Conflict determination } & = \begin{cases}\text { Conflict, } & \text { TTC }<1.5 \mathrm{~s}, \\
\text { Not conflict, } & \text { TTC } \geq 1.5 \mathrm{~s},\end{cases} \\
C_{(t, s)} & =\sum_{j=1}^{N} m\left(\mathrm{TTC}_{j}<1.5\right),
\end{aligned}
$$

where TTC is the time to collision, $x$ is the difference in $x$ coordinate of two vehicles, $y$ is the difference in $y$ coordinate of two vehicles, $V_{s}$ is the subject vehicle speed, $V_{t}$ is the target vehicle speed, $C$ is the number of conflicts, $m$ is the count, $t$ is the time cell, $s$ is the space cell, $j$ is the number of vehicles, and $N$ is the total number of vehicles.

The results of the RORF analysis on the lane change conflict are presented in Figure 5(a) to compare the difference in the lateral conflicts according to the MPR. As a result of the analysis, it was identified that the lateral safety decreases as the MPR increases, contrary to the longitudinal safety. The RORF of MPR $100 \%$ was $29 \%$, which was $56 \%$ 


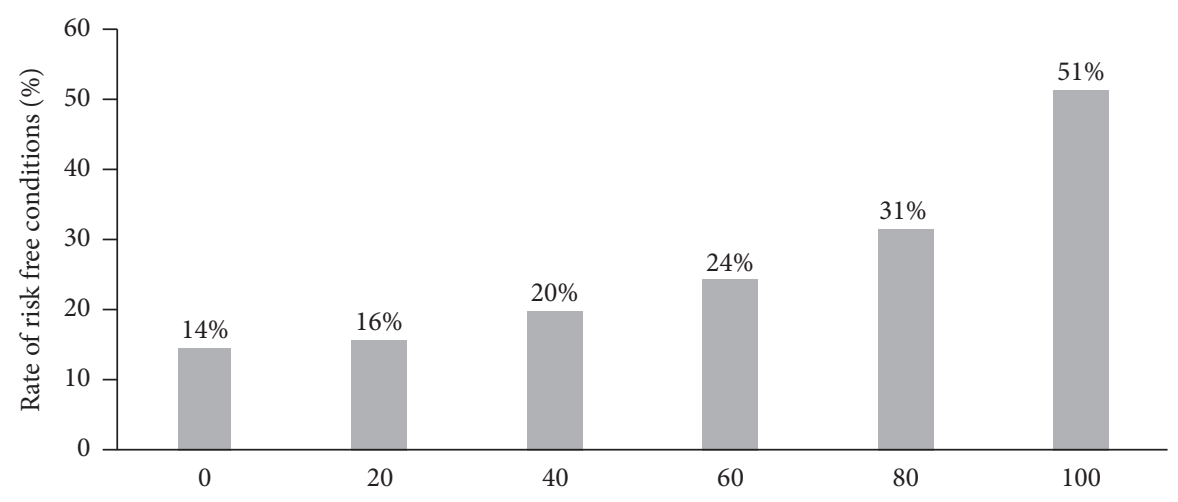

(\%)

(a)
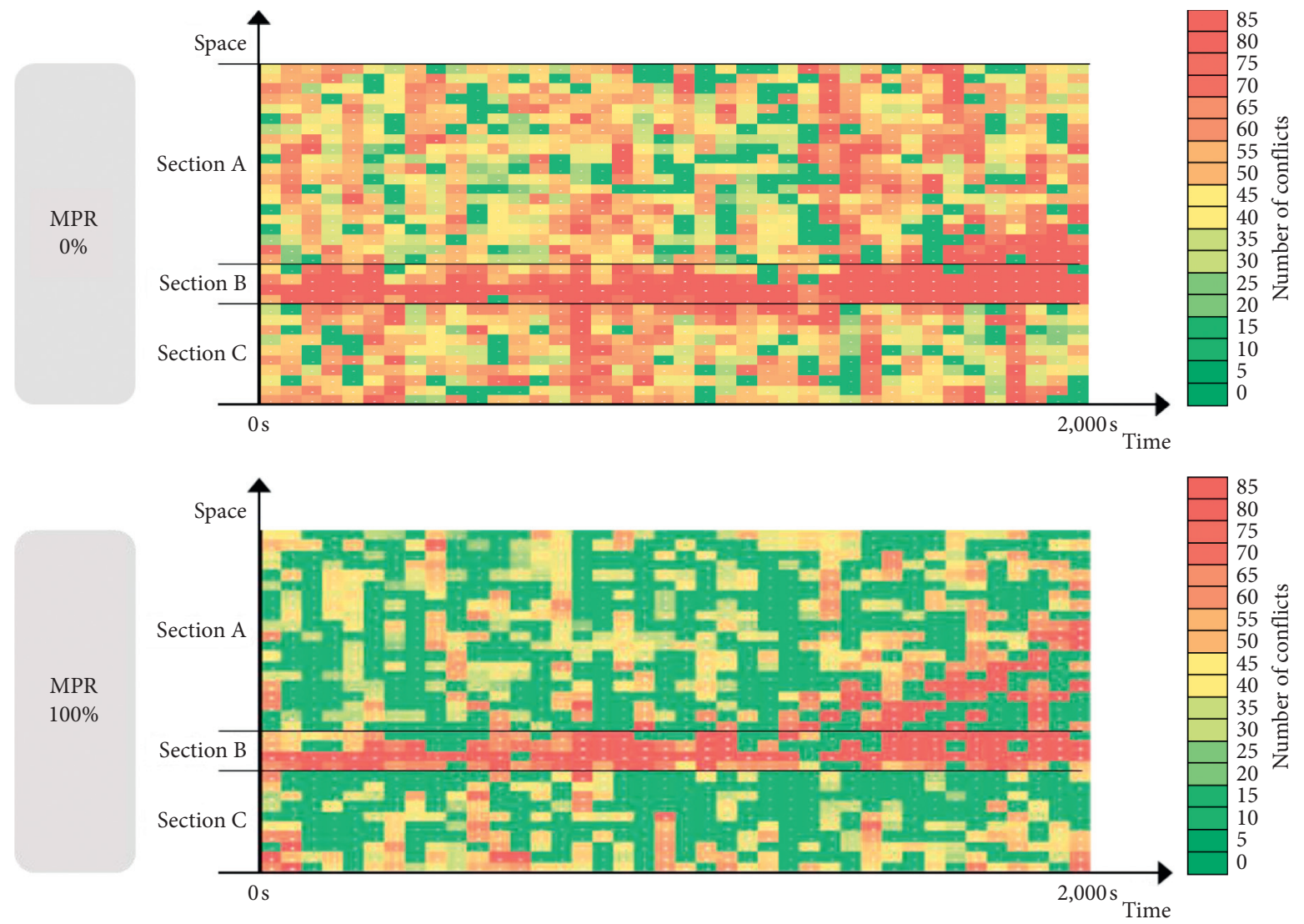

(b)

FIGURE 4: Analysis results of rear-end conflict. (a) Rate of risk-free conditions by MPR and (b) spatiotemporal diagram of conflicts by MPR $0 \%, 100 \%$

lower than that of MPR 0\%. Figure 5(b) shows a comparison of lane change conflicts between MPR $0 \%$ and MPR $100 \%$. In both scenarios, it was found that the number of lane change conflicts in B section is approximately three times as many as in the $\mathrm{A}$ and $\mathrm{C}$ sections. The result of the network safety analysis shows that it is necessary to manage traffic to improve lateral safety rather than longitudinal safety. In addition, a platoon operation strategy should be established to reduce the conflict between the entering vehicle and the platoon in the on-ramp.
3.2.2. Safety Performance on the Rightmost Lane. The safety analyses on the rightmost lane were conducted to evaluate the safety of the truck-designated lane. The speed difference between lanes was used as a surrogate safety measure of the truck-designated lane in the previous study [34]. The speed difference between lanes is obtained by equation (15). An analysis of variance (ANOVA) was performed to clearly identify the speed difference between lanes according to the MPR. The analysis results of the ANOVA are presented in Table 3. To identify the difference in speeds between lanes by 


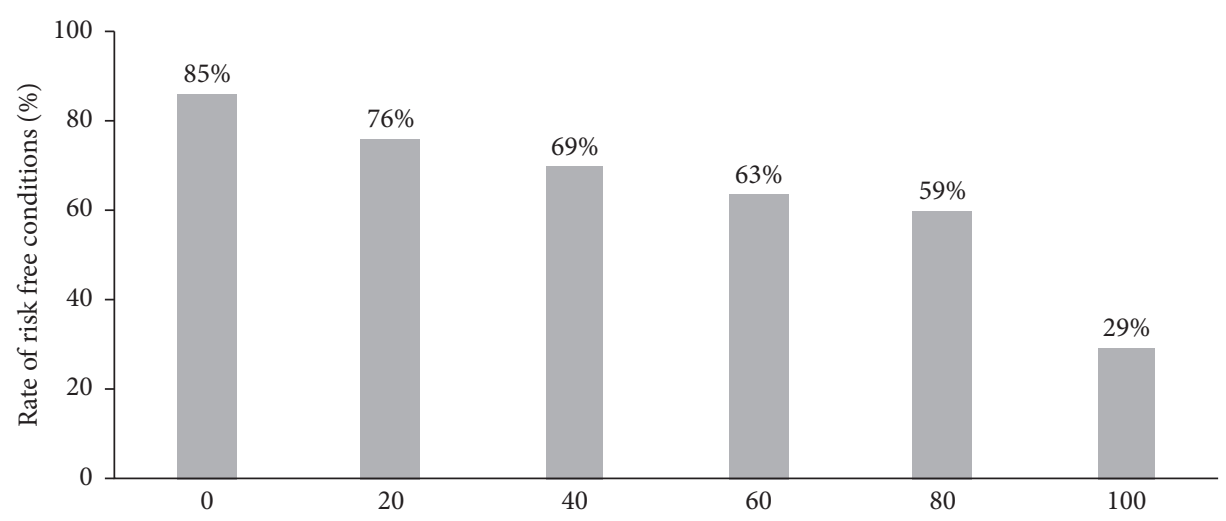

(\%)

(a)
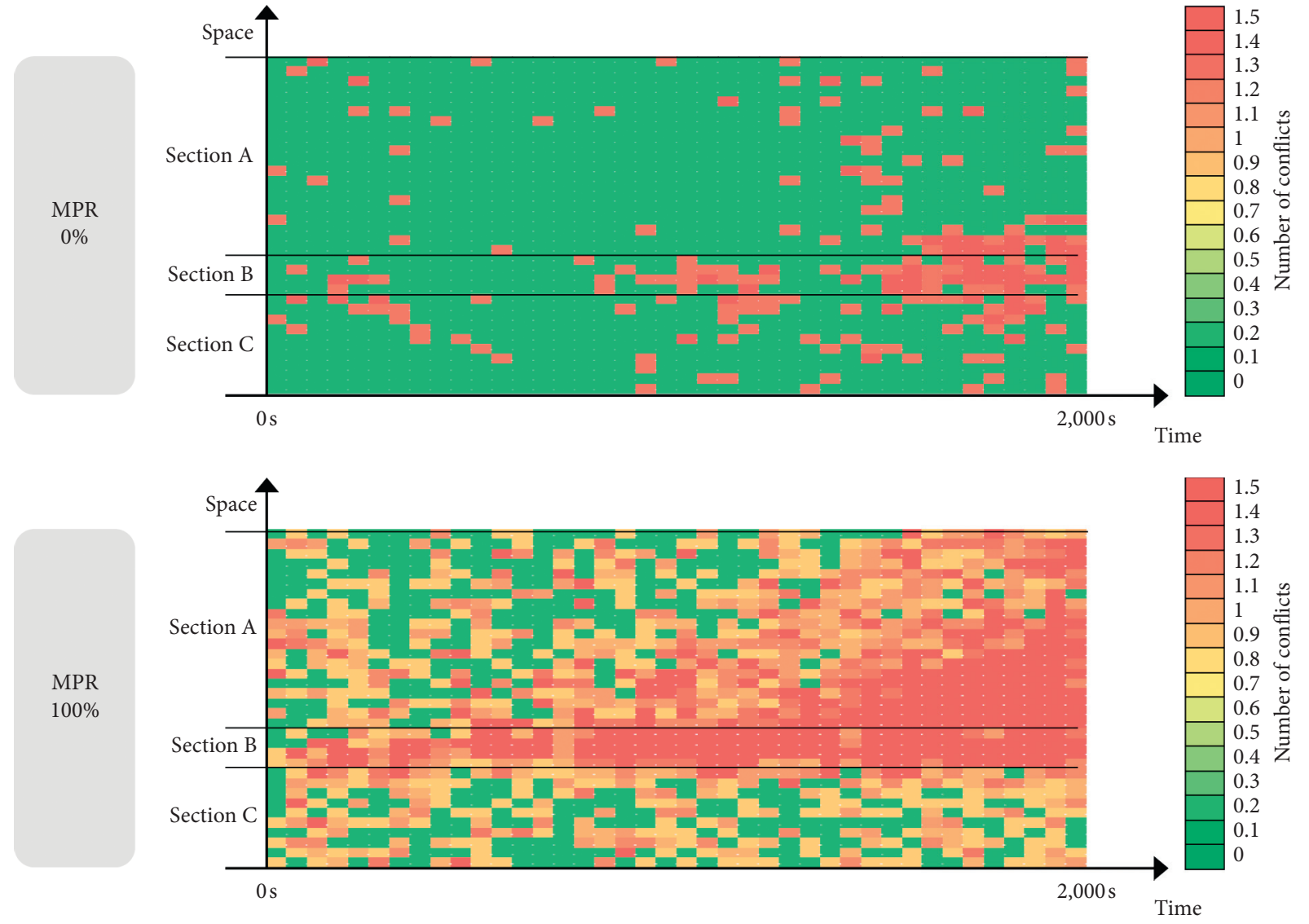

(b)

FIGURE 5: Analysis results of lane change conflict, (a) Rate of risk-free conditions by MPR and (b) spatiotemporal diagram of conflicts by MPR 0\%, 100\%.

MPRs, the ANOVA test was performed, and the analysis results are presented in Table 5. The speed difference between lanes according to the MPR was statistically significant at a 95\% confidence level. In addition, the post hoc analysis was conducted to confirm the significance of the differences between the MPRs. In the MPR 20\% to 
TABLE 5: Analysis results of speed difference between lanes by MPR.

\begin{tabular}{|c|c|c|c|c|c|c|c|c|c|c|c|c|}
\hline \multicolumn{13}{|c|}{ ANOVA } \\
\hline & \multicolumn{2}{|c|}{ Sum of squares } & d.f & Mean square & $F$ & \multicolumn{7}{|c|}{ Sig. } \\
\hline Between groups & 3758.442 & & 5 & 751.688 & 42.821 & & & & & 0.000 & & \\
\hline Within groups & 2527.791 & & 144 & 17.554 & & & & & & & & \\
\hline Total & 6286.234 & & 149 & & & & & & & & & \\
\hline \multicolumn{13}{|l|}{ Post hoc test } \\
\hline \multirow{6}{*}{ Section } & \multicolumn{5}{|c|}{ Subset for alpha $=0.05$} & & 30 & & & & & \\
\hline & & $n$ & 1 & 2 & 3 & & 25 & & & & & \\
\hline & $0 \%$ & 25 & 9.1024 & & & 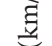 & & & & & & \\
\hline & $20 \%$ & 25 & & 14.1713 & & $\tilde{u}$ & $20-$ & & & & & \\
\hline & $40 \%$ & 25 & & 15.8835 & & & 15 . & & & & & \\
\hline & $80 \%$ & 25 & & 16.4793 & & $\stackrel{\vec{\sharp}}{\mathscr{G}}$ & & & & & & \\
\hline \multirow{4}{*}{ Duncan } & $60 \%$ & 25 & & 17.1070 & & $\stackrel{\theta}{0}$ & $10-$ & & & & & \\
\hline & $100 \%$ & 25 & & & 25.9582 & ֻ̆ & 5 & & & & & \\
\hline & Sig. & & 1.000 & 0.299 & 1.000 & & & 0 & 20 & $40 \quad 60$ & 80 & 100 \\
\hline & & & & & & \multicolumn{7}{|c|}{ MPR (\%) } \\
\hline
\end{tabular}

$80 \%$ scenarios, there was no significant difference in speed difference between lanes. In MPR 100\%, the speed difference between lanes was verified to be approximately $25 \mathrm{~km} / \mathrm{h}$. This is approximately 2.5 times higher than the MPR 0\% speed difference between lanes. The increase in the speed difference between lanes can reduce the stability of the traffic flow and the lane change safety of the entering vehicle in the on-ramp. In the absence of a platoon-only lane, an increase in truck platoons would reduce the mobility and safety of the platoon-designated lane.

$$
\text { speed difference between lanes }=\frac{\left(V_{\text {leftmost lane }}+V_{\text {middle lane }}\right)}{2}-V_{\text {rightmost lane }} \text {, }
$$

where $V$ is the average speed by lane.

\section{Conclusion}

Recent advances in automated vehicle technology are expected to transform roads into productive spaces and lead to exceptional changes in the world. Specifically, platooning, one of the automated driving services is expected to improve traffic mobility and traffic safety. Platooning technology is advancing with the growth of the automated vehicle market and the commercialization of $5 \mathrm{G}$ and C-ITS infrastructures. According to the EU truck platoon roadmap, it is expected that multibrand platooning technology will be possible by 2025. To maximize the effect of platooning, it is necessary to establish a platoon operation strategy in terms of transportation management. In particular, a general vehicle entering an on-ramp must change lanes for merging into the main line. In this situation, a platoon operation strategy should be established so that the general vehicle can change lanes safely. In this study, it is necessary to identify the impact of traffic platooning on the traffic flow when a platoon-only lane is not operational before considering the platoon operation strategy.

The purpose of this study is to identify traffic mobility and safety according to truck platooning on the freeway on-ramp. The platoon formation algorithm was developed to implement the platoon, and the algorithm consisted of the truck sequencing in the platoon, the determination of platoon completion, and the speed control algorithm. The VISSIM COM interface was used to run the proposed algorithm in microscopic traffic simulation environments. The analysis network is a 3-lane freeway with an on-ramp, and the truck drives in the rightmost lane, the designated lane. The main result of the mobility analysis is that as the MPR increases, the mobility of the truck-designated lane, as well as the mobility of the entire network, is reduced. In MPR $100 \%$, the rate of congestion-free condition was $10 \%$ lower than in MPR $0 \%$. The rate of risk-free conditions (ROFC) is defined to identify network safety. Longitudinal safety is improved as the MPR increases, while the lateral safety is reduced. The speed difference between lanes was used as an indicator to conduct a safety analysis of the truck-designated lane. The speed difference between lanes of MPR 100\% was approximately 2.5 times higher than that of MPR $0 \%$.

An automated driving system can create a synergy effect when a traffic management strategy is introduced that considers interactions with general vehicles and automated vehicles. This study is valuable because it explores traffic mobility and safety change through simulation before the introduction of the truck platoon. The following additional studies should be conducted to develop this research into a platoon management strategy. First, the proposed platoon formation algorithm does not include a component to simulate platoon dissipation, which is essential to fully reflect reality. However, a platoon dissolution algorithm that 
reflects car following and gap acceptance conditions should be developed, and a platoon operation strategy should be studied through an analysis of the off-ramp. It is also necessary to ensure that the platoon is formed efficiently, considering the vehicles that can be platooned in the same lane and the surrounding lanes. Second, the scenario of this study considered only the MPR of platoon trucks. However, for the strategy of cluster operation, it is necessary to perform an analysis considering the combination of platoon parameters such as platoon size, intraplatoon spacing, and interplatoon spacing. Further studies should be carried out in consideration of the various vehicle types of the platoon and the operation strategies, such as designated lanes, platoon-only lanes, and high-occupancy toll lanes. In addition, this study used traffic volume corresponding to the level of service (LOS) C, which is the design level of service for freeways [24], as the demand volume in simulation experiments. However, various congestion levels and ratios of trucks in the traffic stream should be taken into consideration in evaluating the impact of truck platooning. Third, we simulated the platoon maneuvering in the VISSIM by adjusting writable parameters such as speed and driving behavior parameters using COM interface. However, it is needed to simulate the behavior of the platoon using an external driver module to completely control the maneuvering of platoons in the simulation. Finally, if the actual maneuvering of automated vehicles is reflected in the simulation, a reliable platoon impact estimation can be obtained. In addition, although this study used safety distance and TTC-based indicators in the safety evaluation by truck platooning, it is necessary to perform the evaluation using a variety of surrogate safety measures.

\section{Data Availability}

The data used to support the findings of this study are available from the corresponding author upon request.

\section{Conflicts of Interest}

The authors declare that there are no conflicts of interest regarding the publication of this article.

\section{Acknowledgments}

This research was supported by a grant from the Transportation and Logistics Research Program, funded by the Ministry of Land, Infrastructure and Transport Affairs of the Korean Government (Project No.: 19TLRP-B101406-05) and also a grant from the Transportation and Logistics Research Program funded by the Ministry of Land, Infrastructure and Transport of the Korea Government (20TLRPB148659-03).

\section{References}

[1] L. Yue, M. Abdel-Aty, Y. Wu, and L. Wang, "Assessment of the safety benefits of vehicles' advanced driver assistance, connectivity and low level automation systems," Accident Analysis \& Prevention, vol. 117, pp. 55-64, 2018.
[2] D. M. Woisetschläger, "Consumer perceptions of automated driving technologies: an examination of use cases and branding strategies," in Autonomous Driving, pp. 687-706, Springer, Berlin, Germany, 2016.

[3] M. S. Rahman, M. Abdel-Aty, J. Lee, and M. H. Rahman, "Safety benefits of arterials' crash risk under connected and automated vehicles," Transportation Research Part C: Emerging Technologies, vol. 100, pp. 354-371, 2019.

[4] L. Cui, J. Hu, and B. B. Park, "Development of a simulation platform for safety impact analysis considering vehicle dynamics, sensor errors, and communication latencies: assessing cooperative adaptive cruise control under cyber attack," Transportation Research Part C: Emerging Technologies, vol. 97, pp. 1-22, 2018.

[5] X. Bujanovic and Y. Yin, "Behaviorally stable vehicle platooning for energy savings," Transportation Research Part C: Emerging Technologies, vol. 99, pp. 37-52, 2019.

[6] A. Duret, M. Wang, and A. Ladino, "A hierarchical approach for splitting truck platoons near network discontinuities," Transportation Research Part B: Methodologica, vol. 132, 2020.

[7] A. Vahidi and A. Sciarretta, "Energy saving potentials of connected and automated vehicles," Transportation Research Part C: Emerging Technologies, vol. 95, pp. 822-843, 2018.

[8] Y. Guo, J. Ma, C. Xiong, X. Li, F. Zhou, and W. Hao, "Joint optimization of vehicle trajectories and intersection controllers with connected automated vehicles: combined dynamic programming and shooting heuristic approach," Transportation Research Part C: Emerging Technologies, vol. 98, pp. 54-72, 2019.

[9] J. Hudson, M. Orviska, and J. Hunady, "People's attitudes to autonomous vehicles," Transportation Research Part A: Policy and Practice, vol. 121, pp. 164-176, 2019.

[10] Y. O. Scherr, B. A. Neumann Saavedra, M. Hewitt, and D. C. Mattfeld, "Service network design with mixed autonomous fleets," Transportation Research Part E: Logistics and Transportation Review, vol. 124, pp. 40-55, 2019.

[11] L. Buckley, S.-A. Kaye, and A. K. Pradhan, "Psychosocial factors associated with intended use of automated vehicles: a simulated driving study," Accident Analysis \& Prevention, vol. 115, pp. 202-208, 2018.

[12] EAMA, "Infographic on EU roadmap for truck platooning," 2019, https://www.acea.be/uploads/publications/Platooning roadmap.pdf.

[13] F. Kitchener, T. English, D. Gopalakrishna et al., "Connected vehicle pilot deployment program phase 2, data management Plan-Wyoming," Publication FHWA-JPO-17-470, FHWA, U.S. Department of Transportation, Washington, DC, USA, 2017.

[14] M. S. Rahman and M. Abdel-Aty, "Longitudinal safety evaluation of connected vehicles' platooning on expressways," Accident Analysis \& Prevention, vol. 117, pp. 381-391, 2018.

[15] S. Lee, C. Oh, and S. Hong, "Exploring lane change safety issues for manually driven vehicles in vehicle platooning environments," IET Intelligent Transport Systems, vol. 12, no. 9, pp. 1142-1147, 2018.

[16] E. Jeong, C. Oh, and S. Lee, "Is vehicle automation enough to prevent crashes? Role of traffic operations in automated driving environments for traffic safety," Accident Analysis \& Prevention, vol. 104, pp. 115-124, 2017.

[17] A. Papadoulis, M. Quddus, and M. Imprialou, "Evaluating the safety impact of connected and autonomous vehicles on motorways," Accident Analysis \& Prevention, vol. 124, pp. 12-22, 2019. 
[18] B. Zhang, E. S. Wilschut, D. M. C. Willemsen, and M. H. Martens, "Transitions to manual control from highly automated driving in non-critical truck platooning scenarios," Transportation Research Part F: Traffic Psychology and Behaviour, vol. 64, pp. 84-97, 2019.

[19] S. C. Calvert, W. J. Schakel, and B. van Arem, "Evaluation and modelling of the traffic flow effects of truck platooning," Transportation Research Part C: Emerging Technologies, vol. 105, pp. 1-22, 2019.

[20] M. Wang, S. van Maarseveen, R. Happee, O. Tool, and B. van Arem, "Benefits and risks of truck platooning on freeway operations near entrance ramp," Transportation Research Record: Journal of the Transportation Research Board, vol. 2673, no. 8, pp. 588-602, 2019.

[21] L. Xiao, M. Wang, and B. van Arem, "Traffic flow impacts of converting an HOV lane into a dedicated CACC lane on a freeway corridor," IEEE Intelligent Transportation Systems Magazine, vol. 12, no. 1, pp. 60-73, 2019.

[22] S. Moon, I. Moon, and K. Yi, "Design, tuning, and evaluation of a full-range adaptive cruise control system with collision avoidance," Control Engineering Practice, vol. 17, no. 4, pp. 442-455, 2009.

[23] C. F. Daganzo and M. J. Cassidy, "Effects of high occupancy vehicle lanes on freeway congestion," Transportation Research Part B: Methodological, vol. 42, no. 10, pp. 861-872, 2008.

[24] Ministry of Land, Transport and Maritime Affairs, Korea Highway Capacity Manual, Ministry of Land, Transport and Maritime Affairs, Sejong, China, 2013.

[25] Ministry of Land, Infrastructure and Transport, Korean Policy for Road Geometry Designs, Ministry of Land, Infrastructure and Transport, Sejong, China, 2013.

[26] M. Amoozadeh, H. Deng, C.-N. Chuah, H. M. Zhang, and D. Ghosal, "Platoon management with cooperative adaptive cruise control enabled by VANET," Vehicular Communications, vol. 2, no. 2, pp. 110-123, 2015.

[27] M. Gouy, K. Wiedemann, A. Stevens, G. Brunett, and N. Reed, "Driving next to automated vehicle platoons: how do short time headways influence non-platoon drivers' longitudinal control?" Transportation Research Part F: Traffic Psychology and Behaviour, vol. 27, pp. 264-273, 2014.

[28] S. Lee and C. Oh, "A methodology to establish operational strategies for truck platoonings on freeway on-ramp areas," Journal of Korean Society of Transportation, vol. 36, no. 2, pp. 67-85, 2018.

[29] E. Brockfeld, R. D. Kühne, and P. Wagner, "Calibration and validation of microscopic traffic flow models," Transportation Research Record: Journal of the Transportation Research Board, vol. 1876, no. 1, pp. 62-70, 2004.

[30] UK Department of Transport, Research on the Impacts of Connected and Autonomous Vehicles (CAVs) on Traffic Flow, UK Department of Transport, London, UK, 2016.

[31] S. Lee, E. Jeong, M. Oh, and C. Oh, "Driving aggressiveness management policy to enhance the performance of mixed traffic conditions in automated driving environments," Transportation Research Part A: Policy and Practice, vol. 121, pp. 136-146, 2019.

[32] D. Gettman, L. Pu, T. Sayed, S. Shelby, and I. Siemens, "Surrogate safety assessment model and validation," Publication FHWA-HRT-08-051, FHWA, U.S. Office of Safety Research and Development, Washington, DC, USA, 2008.

[33] R. Van Der Horst and J. Hogema, "Time-to-collision and collision avoidance systems," Presented at 6th ICTCT Workshop, Austrian Road Safety Board, Salzburg, Austria, 1993.
[34] S. Siuhi and R. Mussa, "Simulation analysis of truck-restricted and high-occupancy vehicle lanes," Transportation Research Record: Journal of the Transportation Research Board, vol. 2012, no. 1, pp. 127-133, 2007. 